\title{
Caracterización clínica y laboratorial de pacientes pediátricos con dengue sin signos de alarma en un hospital de referencia de Paraguay
}

\section{Clinical and laboratory characterization of pediatric patients with dengue without warning signs in a reference hospital in Paraguay}

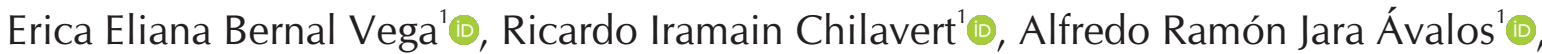 \\ Edith María Rossana Delvalle Acosta',Laura Patricia Arzamendia Alarcón', Landhy \\ Elizabeth Román Almada ${ }^{1}$
}

${ }^{1}$ Universidad Nacional de Asunción, Facultad de Ciencias Médicas, Hospital de Clínicas, Departamento de Emergencias Pediátricas. San Lorenzo, Paraguay.

\section{RESUMEN}

Introducción: El virus del Dengue es transmitido por la picadura de mosquitos hembra de Aedes aegypti. En Paraguay constituye una enfermedad endémica, con epidemias cada 3-5 años, y con diferentes tipos de presentación, desde formas asintomáticas hasta formas graves, que incluso puede desencadenar la muerte. Objetivo: Describir las características clínicas y laboratoriales de pacientes en edad pediátrica con diagnóstico de Dengue sin signos de alarma que acudieron al consultorio del Departamento de Urgencias Pediátricas del Hospital de Clínicas de San Lorenzo, Paraguay, entre noviembre de 2019 y marzo de 2020. Materiales y Métodos: Estudio descriptivo, observacional, retrospectivo, de corte transverso de casos consecutivos. Se incluyeron pacientes de 1 mes a 18 años en un muestreo no probabilístico. Resultados: Se incluyeron 221 pacientes. Se obtuvo una edad promedio de 8,6 años, siendo el 51\% de sexo femenino. En cuanto a la sintomatología, el 100\% de los pacientes manifestaron la presencia de fiebre, seguido en frecuencia por cefalea $(45 \%)$, dolor corporal (36\%) y vómitos (18\%). Se realizó análisis de detección de antígeno NS1 en 107 pacientes, de los cuales 75 resultaron positivos. Conclusión: Se observó una distribución similar en cuanto al sexo, con una mediana de edad de 8 años. Las manifestaciones clínicas más frecuentes fueron fiebre,

\section{ABSTRACT}

Introduction: Dengue virus is transmitted by the bite of female Aedes aegypti mosquitoes. In Paraguay it is an endemic disease, with epidemics every 3-5 years, and with different types of presentation, from asymptomatic to severe forms, which can even trigger death. Objective: To describe the clinical and laboratory characteristics of pediatric patients with a diagnosis of Dengue without warning signs who presented to the Pediatric Emergency Department of the Hospital de Clínicas of San Lorenzo, Paraguay, between November 2019 and March 2020. Materials and Methods: this was a descriptive, observational, retrospective, cross-sectional study of consecutive cases. Patients from 1 month to 18 years of age were included in a non-probability sampling. Results: 221 patients were included. An average age of 8.6 years was obtained, with $51 \%$ being female. Regarding the symptoms, $100 \%$ of the patients manifested the presence of fever, followed in frequency by headache (45\%), body pain $(36 \%)$ and vomiting $(18 \%)$. NS1 antigen detection analysis was performed in 107 patients, of which 75 were positive. Conclusion: A similar gender distribution was observed, with a median age of 8 years. The most frequent clinical manifestations were fever, headache, and body pain; however, a higher frequency of patients with gastrointestinal symptoms was recorded compared to

Correspondencia: Erica Bernal Correo: ericabernalvega@gmail.com

Declaración de conflicto de intereses: Los autores declaran no poseer conflicto de intereses.

Recibido: 03/03/2021 Aceptado: 18/05/2021

DOI: https://doi.org/10.31698/ped.48022021007

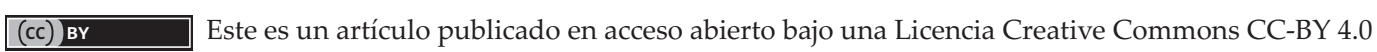


cefalea y dolor corporal, sin embargo, se registró una mayor frecuencia de pacientes con síntomas gastrointestinales respecto a brotes de años anteriores, probablemente relacionado con el serotipo circulante. Se obtuvo un $70 \%$ de positividad de la prueba de antígeno Ns1.

Palabras clave: Fiebre dengue, epidemiología, Paraguay. outbreaks in previous years, probably related to the circulating serotype. The NS1 antigen test was 70\% positive.

Key words: Dengue fever, epidemiology, Paraguay.

\section{INTRODUCCIÓN}

En nuestro país, el Dengue constituye una enfermedad endémica, con epidemias periódicas cada 3 a 5 años, que conlleva una importante morbimortalidad y, por ende, un gran impacto en salud y a nivel socieconómico ${ }^{(1)}$.

Este trabajo tiene como finalidad describir las características clínicas y de laboratorio de los pacientes pediátricos en la última epidemia de Dengue en Paraguay (2019-2020), que han sido clasificados como Dengue sin signos de alarma y, por lo tanto, fueron manejados de manera ambulatoria.

La importancia de este trabajo radica en que, a pesar de ser pacientes estables, el curso de la enfermedad es impredecible ${ }^{(2-5)}$, por lo cual se considera que todos los pacientes son potencialmente de riesgo, y, por tanto, el manejo ambulatorio requiere controles médicos frecuentes, así como la comprensión de los signos de alarma por parte de los padres.

\section{MATERIALES Y MÉTODOS}

Este estudio se llevó a cabo en el Departamento de Urgencias Pediátricas del Hospital de Clínicas entre noviembre de 2019 y marzo de 2020 por medio de datos obtenidos de los registros médicos, los cuales fueron utilizados de manera confidencial, con aprobación del Comité de Ética del Hospital. Se incluyeron pacientes pediátricos con sospecha clínica de Dengue sin signos de alarma, previamente sanos. Un total de 243 pacientes con rango etario entre 1 mes y 18 años fueron inicialmente admitidos en el estudio, cuyo diagnóstico fue Dengue sin signos de alarma, tanto por clínica como por análisis laboratoriales, teniendo en cuenta el contexto del

brote, en que un resultado negativo de NS1 no descarta la enfermedad, y que en epidemias también se considera caso positivo a paciente sintomático y con nexo epidemiológico ${ }^{(2)}$. Los criterios de exclusión fueron coinfecciones (virales o bacterianas) y datos incompletos en las fichas médicas, por lo cual fueron excluidos 8 y 14 pacientes, respectivamente, obteniéndose finalmente un total de 221 pacientes.

Las variables analizadas fueron los datos demográficos (edad, sexo), síntomas (fiebre, cefalea, dolor ocular, mialgias, artralgias, rash, prurito, sangrados, síntomas respiratorios y gastrointestinales) y analítica de laboratorio (determinación de NS1 y serología para Dengue, recuento de hemoglobina/hematocrito, glóbulos blancos y plaquetas, niveles de proteína $\mathrm{C}$ reactiva, hepatograma).

Se realizó análisis estadístico con porcentajes, promedio y mediana, utilizando el programa de Microsoft Excel.

\section{Asuntos éticos}

El trabajo fue aprobado por el Comité de Ética del Hospital de Clínicas.

\section{RESULTADOS}

Se incluyeron 221 pacientes. Se obtuvo una edad promedio de 8,6 años, con una mediana de 8 , siendo el 51\% (112) de sexo femenino y el 49\% (109) de sexo masculino (Gráfico 1).

En cuanto a los síntomas registrados, el 100\% de los pacientes manifestaron la presencia de fiebre, 
seguido por cefalea $(45 \%)$, dolor corporal $(36 \%)$, vómitos $(18 \%)$, dolor abdominal $(16 \%)$, rash $(13 \%)$, dolor ocular (12\%), epistaxis (10\%), diarrea $(9,5 \%), y$ menos frecuentes, dolor de garganta $(4,5 \%)$, artralgias $(4 \%)$, tos $(3 \%)$, congestión nasal $(2 \%)$, prurito $(1 \%)$ y náuseas (1\%). (Tabla 1$)$.

Los resultados informados de la prueba para detección de antígeno NS1 se realizó en 107 pacientes, de los cuales 75 resultaron positivos y 32 fueron negativos. En 1 solo paciente se obtuvo serología IgM, que resultó positiva.
Los valores obtenidos en los hemogramas serán detallados según los grupos etarios (Tabla 2).

En cuanto al valor de proteína $C$ reactiva se obtuvo un registro de $17,7 \mathrm{mg} / \mathrm{dl}$ en promedio, GOT 39,4 UI/l, GPT 23,9 UI/l, Fosfatasa alcalina 206,1 UI/l, bilirrubina total $0,31 \mathrm{mg} / \mathrm{dl}$, bilirrubina directa 0,13 $\mathrm{mg} / \mathrm{dl}$ y bilirrubina indirecta $0,18 \mathrm{mg} / \mathrm{dl}$.

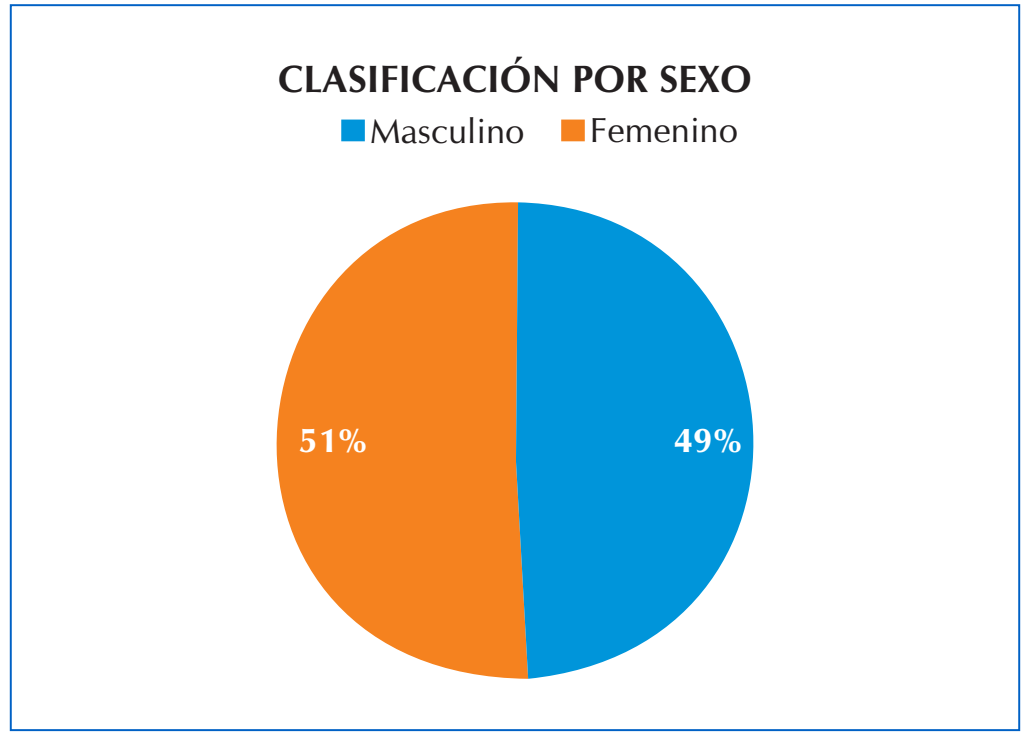

Gráfico 1. Clasificación por sexo $(n=221)$

Tabla 1. Signos y síntomas según rango etario $(n=221)$

\begin{tabular}{lcccc}
\hline \multirow{2}{*}{ Signo/síntoma } & \multicolumn{3}{c}{ Rango Etario } & Total \\
\cline { 2 - 4 } & Lactantes & Preescolares y escolares & Adolescente & \\
\hline Fiebre & 24 & 128 & 69 & $221(100 \%)$ \\
Cefalea & 3 & 58 & 38 & $99(45 \%)$ \\
Dolor corporal & 0 & 40 & 39 & $79(36 \%)$ \\
Vómitos & 4 & 29 & 7 & $40(18 \%)$ \\
Dolor abdominal & 2 & 20 & 13 & $35(16 \%)$ \\
Rash & 5 & 16 & 7 & $28(13 \%)$ \\
Dolor ocular & 1 & 11 & 14 & $26(12 \%)$ \\
Epistaxis & 0 & 19 & 4 & $23(10 \%)$ \\
Diarrea & 5 & 10 & 6 & $21(9,5 \%)$ \\
Dolor de garganta & 1 & 6 & 3 & $10(4,5 \%)$ \\
Artralgias & 0 & 2 & 6 & $8(4 \%)$ \\
Tos & 1 & 4 & 2 & $7(3 \%)$ \\
Congestión nasal & 1 & 2 & 1 & $4(2 \%)$ \\
Prurito & 0 & 1 & 1 & $2(1 \%)$ \\
Náuseas & 0 & 1 & 1 & $2(1 \%)$ \\
\hline
\end{tabular}


Tabla 2. Promedio de resultados de determinaciones del hemograma según el día de enfermedad y los grupos etarios.

\begin{tabular}{|c|c|c|c|c|c|c|c|}
\hline \multicolumn{8}{|c|}{ Lactantes } \\
\hline Día de enfermedad & 1 & 2 & 3 & 4 & 5 & 6 & 7 \\
\hline Hemoglobina (g/dl) & 10,6 & 11,8 & 12 & 11,7 & 12 & 12,1 & 12,2 \\
\hline Hematocrito (\%) & 32 & 34,8 & 35,6 & 34,3 & 35,4 & 35,6 & 35,7 \\
\hline Glóbulos blancos (cél/ $\mu \mathrm{l})$ & 6300 & 5671 & 4417 & 5398 & 5361 & 5398 & 5456 \\
\hline Plaquetas (cél/ $\mu \mathrm{l})$ & 187500 & 223375 & 196167 & 180625 & 177500 & 179430 & 182467 \\
\hline \multicolumn{8}{|c|}{ Preescolares y escolares } \\
\hline Día de enfermedad & 1 & 2 & 3 & 4 & 5 & 6 & 7 \\
\hline Hemoglobina (g/dl) & 12,6 & 12,9 & 12,8 & 12,9 & 12,9 & 12,5 & 12,2 \\
\hline Hematocrito (\%) & 37,9 & 38,6 & 38,2 & 38,4 & 38,3 & 36,6 & 36,4 \\
\hline Glóbulos blancos (cél/ $\mu \mathrm{l})$ & 6381 & 4658 & 4262 & 3871 & 3471 & 3905 & 5054 \\
\hline Plaquetas (cél/ $\mu \mathrm{l})$ & 234667 & 206771 & 198784 & 194979 & 177313 & 186923 & 196231 \\
\hline \multicolumn{8}{|c|}{ Adolescentes } \\
\hline Día de enfermedad & 1 & 2 & 3 & 4 & 5 & 6 & 7 \\
\hline Hemoglobina (g/dl) & 12,6 & 13,6 & 13,7 & 14,2 & 14,1 & 14,2 & 13,8 \\
\hline Hematocrito (\%) & 40,7 & 40,7 & 40,7 & 42 & 40,7 & 41,9 & 41,5 \\
\hline Glóbulos blancos (cél/ $\mu \mathrm{l})$ & 4747 & 4736 & 3931 & 3449 & 3502 & 3646 & 4225 \\
\hline Plaquetas (cél/ $\mu \mathrm{l})$ & 206267 & 193154 & 184385 & 169067 & 162053 & 162545 & 178000 \\
\hline
\end{tabular}

\section{DISCUSIÓN}

En la última epidemia de Dengue del Paraguay (2019-2020), los pacientes que consultaron en el Departamento de Urgencias Pediátricas del Hospital de Clínicas con diagnóstico de Dengue sin signos de alarma, mostraron una distribución por sexo similar con $51 \%$ del sexo femenino y $49 \%$ del masculino, contrario al estudio realizado por Kumar et al. ${ }^{(6)}$, en el que se demostró un mayor predominio del género masculino. La mediana de edad fue de 8 años, a diferencia de estudios previos en que hubo una mayor cantidad de pacientes con menor rango etario, Iramain et al. ${ }^{(7)}$.

En cuanto a los signos y síntomas manifestados, se observó que el $100 \%$ de los pacientes presentaron fiebre, seguido en frecuencia por cefalea $(45 \%)$, dolor corporal (36\%) y síntomas gastrointestinales (vómitos $18 \%$ y dolor abdominal $16 \%$ ), contrario a los hallazgos en el estudio realizado por Iramain et al. ${ }^{(7)}$, que en el año 2018 arrojó una mayor prevalencia de síntomas respiratorios, lo cual podría explicarse por el serotipo circulante predominante (DENV1 en el año 2018 y DENV4 en 2019-2020). En un estudio realizado en Bangladesh en el brote de Dengue del año 2019 por Hasan et al., se observó que la mayoría de los pacientes presentó sintomatología gastrointestinal ${ }^{(8)}$, en el que el serotipo circulante en dicho brote ha sido elDENV3.

En la literatura se menciona la disminución de la cifra de leucocitos como primera anomalía en el hemograma ${ }^{(9)}$, que en el presente trabajo se registró ya a partir del $2^{\circ}$ día de enfermedad en todos los grupos etarios, siendo más marcado en el 3er día de enfermedad en los lactantes y entre el $4^{\circ}$ y $5^{\circ}$ día en pacientes mayores. El valor de las plaquetas mostró el descenso más bajo en el $5^{\circ}$ día de enfermedad en todos los grupos etarios. La concentración sanguínea fue más variable, con valores de hematocrito con mayor aumento promedio entre el $2^{\circ}$ y $4^{\circ}$ día de enfermedad. Cabe recalcar que la totalidad de los pacientes permanecieron en el grupo de Dengue sin signos de alarma, con variaciones en el hemograma que no han influido en la reclasificación de estos. Los descensos de leucocitos y plaquetas son más marcados en pacientes con Dengue con signos de alarma o Dengue grave ${ }^{(10)}$.

Con relación a la proteína $C$ reactiva, se obtuvo un amplio rango de resultados, desde valores negativos 
hasta un máximo de $130 \mathrm{mg} / \mathrm{dl}$, con valor promedio de $17,7 \mathrm{mg} / \mathrm{dl}$. Se constató una leve afectación hepática ${ }^{(11)}$ por aumento de la enzima GOT con promedio de $39,4 \mathrm{mg} / \mathrm{dl}$, sin otras anormalidades en el perfil hepático.

\section{Limitaciones del estudio}

En el Hospital de Clínicas no contamos permanentemente con reactivos para la detección del antígeno NS1 ni serología para Dengue y el costo de estos es elevado en laboratorios privados, por lo cual no fue posible realizar dichas determinaciones en la mayoría de los pacientes.

Otra limitación del estudio es que los datos son recabados de registros médicos y en ocasiones no son completados en su totalidad, con la consecuente disminución del número de participantes.

\section{CONCLUSIÓN}

En este estudio realizado durante el brote de Dengue del 2019-2020, se obtuvo una similitud en cuanto a la distribución por sexo, con una mediana de edad de 8 años, un $70 \%$ de positividad de la prueba de antígeno NS1 ( $\mathrm{n}=107)$ y se registró un aumento de la concentración sanguínea entre el $2^{\circ}$ y $4^{\circ}$ día de enfermedad, con un descenso de leucocitos entre el 3er y $5^{\circ}$ día y las plaquetas en el $5^{\circ}$ día de enfermedad. En este grupo de pacientes no se observó afectación hepática.

Se observó una mayor frecuencia de síntomas gastrointestinales con respecto a epidemias de años anteriores, lo cual se debe tener en cuenta para una sospecha diagnóstica precoz y un manejo adecuado de los pacientes.

\section{Contribución de autoría}

Todos los mencionados han cumplido con los 4 items de autoría:

1. Contribuciones sustanciales a la concepción o diseño del trabajo; o la adquisición, análisis o interpretación de datos para el trabajo; $Y$

2. Redactar el trabajo o revisarlo críticamente en busca de contenido intelectual importante; $Y$

\section{Aprobación final de la versión a publicar}

4. Acuerdo de ser responsable de todos los aspectos del trabajo para garantizar que las cuestiones relacionadas con la precisión o integridad de cualquier parte del trabajo se investiguen y resuelvan adecuadamente.

\section{REFERENCIAS}

1. Martínez C, Arbo A. Características epidemiológicas del dengue en niños menores de 15 años en el Paraguay. 20082013. Rev del Inst Med Trop. 2016;11(2):4-14. doi: https://doi.org/10.18004/imt/20161124-14

2. Ministerio de Salud pública y Bienestar Social, Organización Panamericana de la Salud. Dengue: Guía de manejo clínico. Asunción: OPS; 2012.

3. Kumar R, Sekhar G, N A, M K. Clinical profile, laboratory investigations and outcome in dengue positive children in south India. Int J Res Pharm Sci. 2020;11(2):17580. doi: https://doi.org/10.26452/ijrps.v11iSPL2.2197
4. Izquierdo Estevez A, Martínez Torrez E. Utilidad de la identificación de los signos de alarma en niños y adolescentes con dengue. Rev Cubana Pediatr. 2019;91(2):e644.

5. Martínez-Cuellar C, Lovera D, Galeano F, Gatti L, Arbo A. Non-structural protein 1 (NS1) of Dengue virus detection correlates with severity in primary but not in secondary Dengue infection. J Clin Virol. 2020;124:104259. doi: https://doi.org/10.1016/j.jcv.2020.104259

6. Kumar R et al. Dengue in children and young adults, a cross-sectional study from the western part of Uttar Pradesh. J Fam Med Prim Care. 2020;9:293-7. doi: https://doi.org/10.4103/jfmpc.jfmpc_770_19 
7. Iramain R, Jara A, Bogado N, Cardozo L, Morinigo M, De Jesus R. Clinical and Laboratory Behavior of Dengue in Children Hospitalized in 2 Epidemics. Period 2012 - 2013 and 2017 - 2018. EC Paediatrics 8.4 (2019): 250-257.

8. Hasan M, Tabassum T, Sharif M, Khan M, Bipasha A, Basher A. Clinico-epidemiologic characteristics of the 2019 dengue outbreak in Bangladesh. Trans R Soc Trop Med Hyg. 2021;115(7):733-740. doi: https://doi.org/10.1093 /trstmh/traa126

9. Organización Panamericana de la Salud. Dengue. Guía para la atención de enfermos en la región de las Américas. Washington: OPS; 2016.
10. Jayarajah U, Madarasinghe M, Hapugoda D, Dissanayake U, Perera L, Kannangara, et al. Clinical and Biochemical Characteristics of Dengue Infections in Children From Sri Lanka. Glob Pediatr Health. 2020;7:2333794X20974207. doi: https://doi.org/10.1177/2333794X20974207

11. Prasad D, Bhriguvanshi A. Clinical Profile, Liver Dysfunction and Outcome of Dengue Infection in Children a prospective observational study. Pediatr Infect Dis J. 2020;39(2):97-101. doi: https://doi.org/10.1097/IN F.0000000000002519 